\title{
Adomian Decomposition Method for Bernoulli Differential Equations
}

\author{
E. U. Agom ${ }^{1}$, F. O. Ogunfiditimi ${ }^{2}$ \\ ${ }^{1}$ Department of Mathematics University of Calabar, Calabar, Nigeria \\ ${ }^{2}$ Department of Mathematics, University of Abuja, Abuja, Nigeria
}

\begin{abstract}
In this paper, Adomoian decomposition method is applied to Bernoulli differential equations and the general Adomian polynomial for this class of equation is presented. Different Bernoulli differential equations with variable exponent of the nonlinear terms were considered as test problems. And the result shows fantastic exact solutions as those obtained by classical method.
\end{abstract}

Keywords: Adomian Decomposition Method, Bernoulli Differential equations.

\section{Introduction}

Bernoulli differential equations (BDE) are nonlinear differential equations named after J. Bernoulli, a swiss scientist. There are used in modern Physics for modeling the dynamics behind certain circuit elements known as Bernoulli memristors. These types of differential equations are special because they are nonlinear with exact solutions. The equation has a nonlinear term which is a function of the independent variable raise to a certain exponent, say $\mathrm{n}$. When $\mathrm{n}$ is zero or one, the $\mathrm{BDE}$ is linear. But for $\mathrm{n} \geq 2$, substitution is carried out to transform it to a linear form which can then be solved linearly [6] and [7]. In this paper we apply Adomian decomposition method (ADM) to solve BDE with $\mathrm{n} \geq 2$.

Adomian Decomposition Method (ADM) generates a solution inform of a series whose terms are determined by a recursive relation using the Adomian polynomial, [1], [8] and [2]. Since it was presented in 1980's several modification of the Adomian polynomial has been presented [4]. [5] introduced a modified Adomian polynomial which converges slightly faster than the original and also presented accelerated Adomian polynomial. Despite various types of Adomian polynomial available, the original Adomian polynomial is generally being used based on the advantage of convenient algorithm which is easily remembered.

\section{Description ADM for BDE}

The BDE is given as

$$
\frac{\mathrm{d} \theta}{\mathrm{dt}}+\mathrm{p}(\mathrm{t}) \theta=\mathrm{f}(\mathrm{t}) \theta^{\varphi}
$$

Here we consider a case of $\varphi \geq 2$ and we use the ADM to find solution to equation (1). The ADM consist of approximating the solution of (1) as an infinite series

$$
\theta=\sum_{\mathrm{n}=0}^{\infty} \theta_{\mathrm{n}}
$$

and decomposing the nonlinear operator $\mathrm{N}(\theta)=\theta^{\varphi}$ as

$$
\mathrm{N}(\theta)=\sum_{\mathrm{n}=0}^{\infty} \mathrm{A}_{\mathrm{n}}
$$

where $A_{n}$ is Adomian polynomial, see [1]-[5], given by

$$
\mathrm{A}_{\mathrm{n}}=\frac{1}{\mathrm{n} !} \mathrm{D}^{(\mathrm{n})}\left[\mathrm{N}\left(\sum_{\mathrm{i}=0}^{\infty} \phi^{\mathrm{i}} \theta_{\mathrm{i}}\right)\right]
$$

where $D^{(n)}=\frac{d^{n}}{d \phi^{n}}$ and $\phi$ is a grouping parameter. In operator form equation (1) is given as

$$
\mathrm{L} \theta=-\mathrm{P}(\mathrm{t}) \theta+\mathrm{f}(\mathrm{t}) \theta^{\varphi}
$$

where $\mathrm{L}=\frac{\mathrm{d}}{\mathrm{dt}}$ is a differential operator. Taking inverse linear operator $\mathrm{L}^{-1}$ on both sides of equation (5), we have

$$
\theta(\mathrm{t})-\mathrm{a}(\mathrm{t})=-\mathrm{L}^{-1}[\mathrm{p}(\mathrm{t}) \theta]+\mathrm{L}^{-1}\left[\mathrm{f}(\mathrm{t}) \mathrm{A}_{\mathrm{n}}\right]
$$

For BDE $\mathrm{L}^{-1}=\mathrm{f}(.) \mathrm{dt}$ Applying equation (2), (3) and (4) in (6), we obtain

$$
\sum_{n=0}^{\infty} \theta_{n}=a(t)-L^{-1}\left[p(t) \sum_{n=0}^{\infty} \theta_{n}\right]+L^{-1}\left[f(t) A_{n}\right]
$$

where the recursive relation is given as

$$
\begin{aligned}
& \theta_{0}(t)=a(t) \\
& \theta_{n+1}(t)=-L^{-1}\left[p(t) \sum_{n=0}^{\infty} \theta_{n}\right]+L^{-1}\left[f(t) A_{n}\right]
\end{aligned}
$$

The first seven Adomian polynomial of BDE from equation (4) are given as

$$
\begin{aligned}
\mathrm{A}_{0} & =\theta_{0}^{\varphi} \\
\mathrm{A}_{1} & =\varphi \theta_{0}^{\varphi-1} \theta_{1} \\
\mathrm{~A}_{2} & =\frac{1}{2} \varphi(\varphi-1) \theta_{0}^{\varphi-2} \theta_{1}^{2}+\varphi \theta_{0}^{\varphi-1} \theta_{2} \\
\mathrm{~A}_{3} & =\frac{1}{6} \varphi(\varphi-1)(\varphi-2) \theta_{0}^{\varphi-3} \theta_{1}^{3}+\varphi(\varphi-1) \theta_{0}^{\varphi-2} \theta_{1} \theta_{2} \\
& +\varphi \theta_{0}^{\varphi-1} \theta_{3} \\
\mathrm{~A}_{4} & =\frac{1}{24} \varphi(\varphi-1)(\varphi-2)(\varphi-3) \theta_{0}^{\varphi-4} \theta_{1}^{4} \\
& +\frac{1}{2} \varphi(\varphi-1)(\varphi-2) \theta_{0}^{\varphi-3} \theta_{1}^{2} \theta_{2}
\end{aligned}
$$




\section{International Journal of Science and Research (IJSR)}

ISSN (Online): 2319-7064

Index Copernicus Value (2013): 6.14 | Impact Factor (2014): 5.611

$$
\begin{aligned}
& +\frac{1}{2} \varphi(\varphi-1) \theta_{0}^{\varphi-2} \theta_{2}^{2}+\varphi(\varphi-1) \theta_{0}^{\varphi-2} \theta_{1} \theta_{3} \\
& +\varphi \theta_{0}^{\varphi-1} \theta_{4} \\
& A_{5}=\frac{1}{120} \varphi(\varphi-1)(\varphi-2)(\varphi-3)(\varphi-4) \theta_{0}^{\varphi-5} \theta_{1}^{5} \\
& +\frac{1}{6} \varphi(\varphi-1)(\varphi-2)(\varphi-3) \theta_{0}^{\varphi-4} \theta_{1}^{3} \theta_{2} \\
& +\frac{1}{2} \varphi(\varphi-1)(\varphi-2) \theta_{0}^{\varphi-3} \theta_{1} \theta_{2}^{2} \\
& +\frac{1}{2} \varphi(\varphi-1)(\varphi-2) \theta_{0}^{\varphi-3} \theta_{1}^{2} \theta_{3}++\varphi(\varphi-1) \theta_{0}^{\varphi-2} \theta_{2} \theta_{3} \\
& +\varphi(\varphi-1) \theta_{0}^{\varphi-2} \theta_{1} \theta_{4}+\varphi \theta_{0}^{\varphi-1} \theta_{5} \\
& \mathrm{~A}_{6}=\frac{1}{720} \varphi(\varphi-1)(\varphi-2)(\varphi-3)(\varphi-4)(\varphi-5) \theta_{0}^{\varphi-6} \theta_{1}^{6} \\
& +\frac{1}{24} \varphi(\varphi-1)(\varphi-2)(\varphi-3)(\varphi-4) \theta_{0}^{\varphi-5} \theta_{1}^{4} \theta_{2} \\
& +\frac{1}{4} \varphi(\varphi-1)(\varphi-2)(\varphi-3) \theta_{0}^{\varphi-4} \theta_{1}^{2} \theta_{2}^{2} \\
& +\frac{1}{6} \varphi(\varphi-1)(\varphi-2)(\varphi-3) \theta_{0}^{\varphi-4} \theta_{1}^{3} \theta_{3} \\
& +\frac{1}{6} \varphi(\varphi-1)(\varphi-2) \theta_{0}^{\varphi-3} \theta_{2}^{3} \\
& +\varphi(\varphi-1)(\varphi-2) \theta_{0}^{\varphi-3} \theta_{1} \theta_{2} \theta_{3} \\
& +\frac{1}{2} \varphi(\varphi-1)(\varphi-2) \theta_{0}^{\varphi-3} \theta_{1}^{2} \theta_{4}+\frac{1}{2} \varphi(\varphi-1) \theta_{0}^{\varphi-2} \theta_{3}^{2} \\
& +\varphi(\varphi-1) \theta_{0}^{\varphi-2} \theta_{2} \theta_{2}+\varphi(\varphi-1) \theta_{0}^{\varphi-2} \theta_{1} \theta_{5} \\
& +\varphi \theta_{0}^{\varphi-1} \theta_{6}
\end{aligned}
$$

\section{Illustrative Examples}

In this section, the ADM for BDE described earlier will be demonstrated on two examples. And we compare the analytical solution with those obtained by ADM.

\section{Example 1}

Consider

$$
\theta^{\prime}-2 \theta-\theta^{5}=0, \quad \theta(0)=1,
$$

The exact solution of equation (10) is given as

$$
\theta^{4}=\frac{2}{3 \mathrm{e}^{-8 \mathrm{t}}-1}
$$

In series form, equation (11) is given as

$$
\theta=1+3 \mathrm{t}+\frac{21}{2} \mathrm{t}^{2}+\frac{109}{2} \mathrm{t}^{3}+\frac{2563}{8} \mathrm{t}^{4}+\mathrm{M}
$$

where

$$
\begin{aligned}
\mathrm{M}= & \frac{78841}{40} \mathrm{t}^{5}+\frac{3000607}{240} \mathrm{t}^{6}+\frac{136150309}{1680} \mathrm{t}^{7} \\
& +\frac{1024596109}{1920} \mathrm{t}^{8}+\frac{43015477461}{120960} \mathrm{t}^{9}
\end{aligned}
$$

$$
+\frac{289361431 \Theta 207}{1209600} \mathrm{t}^{10} \ldots
$$

Applying equation (8) and (9) to equation (10), we obtained $\theta_{0}=1$

$$
\theta_{1}=\int_{0}^{\mathrm{t}}\left(2 \theta_{0}+\theta_{0}^{5}\right) \mathrm{dt}=3 \mathrm{t}
$$$$
\theta_{2}=\int_{0}^{\mathrm{t}}\left(2 \theta_{1}+5 \theta_{1} \theta_{0}^{4}\right) \mathrm{dt}=\frac{21}{2} \mathrm{t}^{2}
$$$$
\theta_{3}=\int_{0}^{\mathrm{t}}\left(2 \theta_{2}+5 \theta_{2} \theta_{0}^{4}+10 \theta_{1}^{2} \theta_{0}^{3}\right) \mathrm{dt}=\frac{109}{2} \mathrm{t}^{3}
$$$$
\theta_{4}=\int_{0}^{\mathrm{t}}\left(2 \theta_{3}+5 \theta_{3} \theta_{0}^{4}+20 \theta_{2} \theta_{1} \theta_{0}^{3}+10 \theta_{1}^{3} \theta_{0}^{2}\right) \mathrm{dt}=\frac{2563}{8} \mathrm{t}^{4}
$$$$
\theta_{5}=\int_{0}^{\mathrm{t}}\left(2 \theta_{4}+5 \theta_{4} \theta_{0}^{4}+20 \theta_{3} \theta_{1} \theta_{0}^{3}+10 \theta_{2}^{2} \theta_{0}^{3}\right.
$$$$
\left.+30 \theta_{2} \theta_{1}^{2} \theta_{0}^{2}+5 \theta_{1}^{4} \theta_{0}\right) \mathrm{dt}=\frac{78841}{40} \mathrm{t}^{5}
$$$$
\theta_{6}=\int_{0}^{t}\left(2 \theta_{5}+5 \theta_{5} \theta_{0}^{4}+20 \theta_{4} \theta_{1} \theta_{0}^{3}+20 \theta_{3} \theta_{2} \theta_{0}^{3}\right.
$$$$
\left.+30 \theta_{3} \theta_{1}^{2} \theta_{0}^{2}+30 \theta_{3} \theta_{2}^{2} \theta_{1} \theta_{0}^{2}+20 \theta_{2} \theta_{1}^{3} \theta_{0}+\theta_{1}^{5}\right) \mathrm{dt}
$$$$
=\frac{3000607}{240} \mathrm{t}^{6}
$$

$$
\begin{aligned}
\theta_{7}=\int_{0}^{\mathrm{t}} & \left(2 \theta_{6}+5 \theta_{2} \theta_{1}^{4}+60 \theta_{3} \theta_{2} \theta_{1} \theta_{0}^{2}+20 \theta_{5} \theta_{1} \theta_{0}^{3}\right. \\
& +20 \theta_{2} \theta_{4} \theta_{0}^{3}+30 \theta_{4} \theta_{0}^{2} \theta_{1}^{2}+30 \theta_{2}^{2} \theta_{0} \theta_{1}^{2} \\
& \left.+20 \theta_{3} \theta_{0} \theta_{1}^{3}+10 \theta_{2}^{3} \theta_{0}^{2}+10 \theta_{0}^{3} \theta_{3}^{2}+5 \theta_{0}^{4} \theta_{6}\right) \mathrm{dt} \\
= & \frac{136150309}{1680} \mathrm{t}^{7}
\end{aligned}
$$

$\theta_{0}, \theta_{1}, \ldots \theta_{7}$ obtained by ADM are the same as the first 8 terms of equation (12) which is the exact solution of BDE (10). Figure 1 and 2 further shows the similarities between results obtained by ADM and that of exact solution. In Figure 1, we considered an infinite series of $\theta$ while in Figure 2 we considered $\theta=\sum_{\mathrm{n}=0}^{7} \theta$.

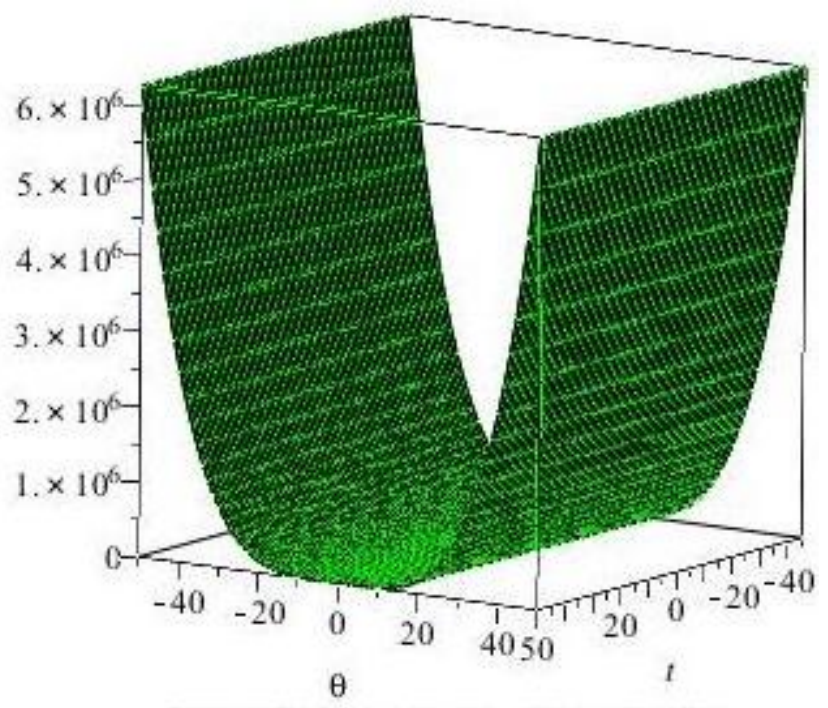

Figure 1: Ex act solution of equation (10) 


\section{International Journal of Science and Research (IJSR) \\ ISSN (Online): 2319-7064}

Index Copernicus Value (2013): 6.14 | Impact Factor (2014): 5.611

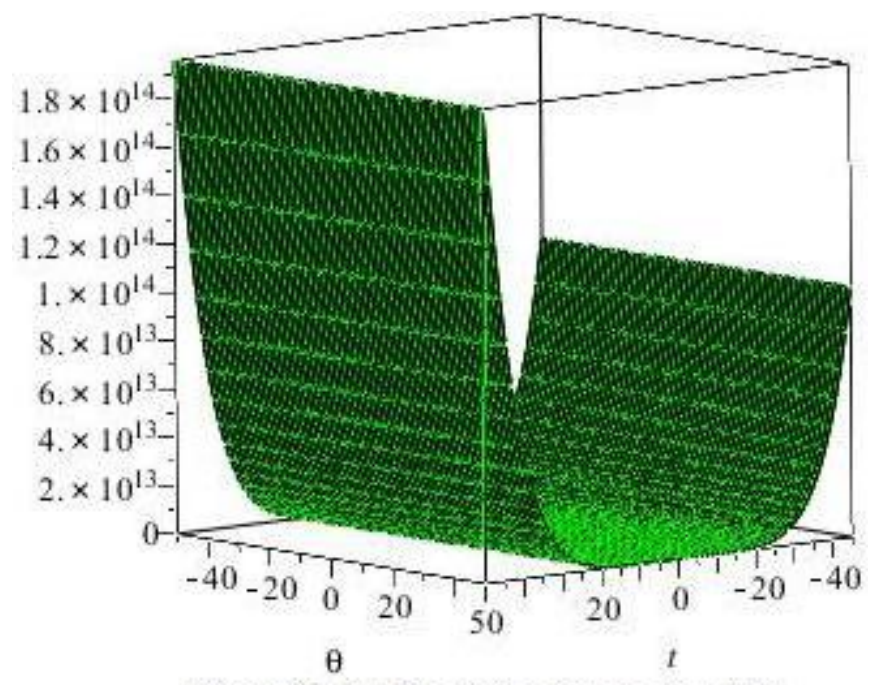

Figure 2: ADM solution of equation (10)

\section{Example 2.}

Consider

$$
\theta^{\prime}-\theta=\mathrm{t} \theta^{6}, \quad \theta(0)=1
$$

The exact solution of equation (13) is given as

$$
\theta^{5}=\frac{5 \mathrm{e}^{5 \mathrm{t}}}{\mathrm{e}^{5 \mathrm{t}}-5 \mathrm{t} \mathrm{e}^{5 \mathrm{t}}+4}
$$

In series form, equation (14) is given as

$$
\theta=1+\mathrm{t}+\mathrm{t}^{2}+\frac{7}{3} \mathrm{t}^{3}+\frac{35}{6} \mathrm{t}^{4}+\frac{419}{30} \mathrm{t}^{5}+\mathfrak{I}
$$

where

$$
\mathfrak{I}=\frac{6269}{180} \mathrm{t}^{6}+\frac{113333}{1260} \mathrm{t}^{7}+\frac{2375831}{10080} \mathrm{t}^{8}
$$

Similarly, applying equation (8) and (9) to equation (10), we have

$$
\begin{aligned}
\theta_{0} & =1 \\
\theta_{1} & =\int_{0}^{\mathrm{t}}\left(\theta_{0}+\mathrm{t} \theta_{0}^{6}\right) \mathrm{dt}=\mathrm{t}+\frac{1}{2} \mathrm{t}^{2} \\
\theta_{2} & =\int_{0}^{\mathrm{t}}\left(\theta_{1}+6 \mathrm{t} \theta_{1} \theta_{0}^{5}\right) \mathrm{dt}=\frac{3}{4} \mathrm{t}^{4}+\frac{13}{6} \mathrm{t}^{3}+\frac{1}{2} \mathrm{t}^{2} \\
\theta_{3} & =\int_{0}^{\mathrm{t}}\left(\theta_{2}+\mathrm{t}\left(6 \theta_{2} \theta_{0}^{5}+15 \theta_{1}^{2} \theta_{0}^{5}\right)\right) \mathrm{dt} \\
& =\frac{11}{8} \mathrm{t}^{6}+\frac{23}{4} \mathrm{t}^{5}+\frac{121}{24} \mathrm{t}^{4}+\frac{1}{6} \mathrm{t}^{3} \\
\theta_{4} & =\int_{0}^{\mathrm{t}}\left(\theta_{3}+\mathrm{t}\left(6 \theta_{3} \theta_{0}^{5}+30 \theta_{2} \theta_{1} \theta_{0}^{4}+20 \theta_{1}^{3} \theta_{0}^{3}\right)\right) \mathrm{dt} \\
& =\frac{11}{4} \mathrm{t}^{8}+\frac{121}{8} \mathrm{t}^{7}+\frac{277}{12} \mathrm{t}^{6}+\frac{197}{24} \mathrm{t}^{5}+\frac{1}{24} \mathrm{t}^{4} \\
\theta_{5} & =\int_{0}^{\mathrm{t}}\left(\theta_{4}+\mathrm{t}\left(24 \theta_{4} \theta_{0}^{5}+120 \theta_{3} \theta_{1} \theta_{0}^{4}+60 \theta_{2}^{2} \theta_{0}^{4}\right.\right. \\
& \left.\left.+240 \theta_{2} \theta_{1}^{2} \theta_{0}^{3}+60 \theta_{1}^{4} \theta_{0}^{2}\right)\right) \mathrm{dt} \\
& =\frac{231}{10} \mathrm{t}^{10}+\frac{627}{4} \mathrm{t}^{9}+\frac{64999}{192} \mathrm{t}^{8}+\frac{20701}{84} \mathrm{t}^{7} \\
& +\frac{5381}{144} \mathrm{t}^{6}+\frac{1}{120} \mathrm{t}^{5} \\
\theta_{6} & =\int_{0}^{\mathrm{t}}\left(\theta_{5}+\mathrm{t}\left(6 \theta_{5} \theta_{0}^{5}+30 \theta_{4} \theta_{1} \theta_{0}^{4}+30 \theta_{3} \theta_{2} \theta_{0}^{4}\right.\right.
\end{aligned}
$$

$$
\begin{aligned}
& \left.\left.+60 \theta_{3} \theta_{1}^{2} \theta_{0}^{3}+60 \theta_{1} \theta_{2}^{2} \theta_{0}^{3}+60 \theta_{2} \theta_{1}^{3} \theta_{0}^{2}+6 \theta_{1}^{5} \theta_{0}\right)\right) \mathrm{dt} \\
& =\frac{847}{40} \mathrm{t}^{12}+\frac{838}{5} \mathrm{t}^{11}+\frac{87043}{192} \mathrm{t}^{10}+\frac{5872849}{12096} \mathrm{t}^{9} \\
& +\frac{80863}{448} \mathrm{t}^{8}+\frac{73561}{5040} \mathrm{t}^{7}+\frac{1}{120} \mathrm{t}^{6} \\
& \theta_{7}=\int_{0}^{\mathrm{t}}\left(\theta_{6}+\mathrm{t}\left(90 \theta_{1}^{2} \theta_{2}^{2} \theta_{0}^{2}+30 \theta_{0} \theta_{2} \theta_{1}^{4}+60 \theta_{0}^{3} \theta_{4} \theta_{1}^{2}\right.\right. \\
& +60 \theta_{3} \theta_{1}^{3} \theta_{0}^{2}+120 \theta_{1} \theta_{3} \theta_{2} \theta_{0}^{3}+30 \theta_{2} \theta_{4} \theta_{0}^{4}+\theta_{1}^{6} \\
& +30 \theta_{0}^{4} \theta_{2} \theta_{4}+30 \theta_{5} \theta_{1} \theta_{0}^{4}+15 \theta_{0}^{4} \theta_{3}^{2} \\
& \left.\left.+20 \theta_{2}^{3} \theta_{0}^{3}+6 \theta_{0}^{5} \theta_{6}\right)\right) \mathrm{dt} \\
& =\frac{7997}{160} \mathrm{t}^{14}+\frac{243911}{520} \mathrm{t}^{13}+\frac{2070129}{1280} \mathrm{t}^{12} \\
& +\frac{331464313}{133056} \mathrm{t}^{11}+\frac{19792177}{12096} \mathrm{t}^{10}+\frac{2278537}{6720} \mathrm{t}^{9} \\
& =\frac{400153}{40320} \mathrm{t}^{8}+\frac{1}{5040} \mathrm{t}^{7} \\
& \theta_{8}=\int_{0}^{\mathrm{t}}\left(\theta_{7}+\mathrm{t}\left(120 \theta_{0}^{3} \theta_{4} \theta_{2} \theta_{1}+180 \theta_{1}^{2} \theta_{3} \theta_{2} \theta_{0}^{2}+6 \theta_{2} \theta_{1}^{5}\right.\right. \\
& +6 \theta_{7} \theta_{0}^{5}+30 \theta_{5} \theta_{2} \theta_{0}^{4}+60 \theta_{5} \theta_{1}^{2} \theta_{0}^{3}+60 \theta_{3} \theta_{2}^{2} \theta_{0}^{3} \\
& +60 \theta_{0} \theta_{2}^{2} \theta_{1}^{3}+30 \theta_{1} \theta_{6} \theta_{0}^{4}+30 \theta_{0} \theta_{3} \theta_{1}^{4}+60 \theta_{1} \theta_{3}^{2} \theta_{0}^{3} \\
& \left.\left.+30 \theta_{3} \theta_{3} \theta_{0}^{4}+60 \theta_{1} \theta_{0}^{2} \theta_{2}^{3}+60 \theta_{4} \theta_{0}^{2} \theta_{1}^{3}\right)\right) \mathrm{dt} \\
& =\frac{19107}{160} \mathrm{t}^{16}+\frac{2659899}{2080} \mathrm{t}^{15}+\frac{306126861}{58240} \mathrm{t}^{14} \\
& +\frac{11955451537}{11531520} \mathrm{t}^{13}+\frac{1596168476}{1596672} \mathrm{t}^{10} \\
& +\frac{257334193}{60480} \mathrm{t}^{11}+\frac{983897}{1680} \mathrm{t}^{10}+\frac{2639641}{362880} \mathrm{t}^{9} \\
& +\frac{1}{40320} \mathrm{t}^{8} \\
& \sum_{n=0}^{8} \theta_{n}=1+t+t^{2}+\frac{7}{3} t^{3}+\frac{35}{6} t^{4}+\frac{419}{30} t^{5}+\ldots
\end{aligned}
$$

All the terms of equation (16) are the same as those of equation (15), which is the classical solution of BDE (13). Thus, more calculation of the terms of $\theta_{\mathrm{n}}$ gives more accurate results. Hence, the required accuracy by ADM can be enhanced by inclusion of more terms of $\theta_{n}$. Figures 3 and 4 further shows that there is no difference between ADM and that of exact solution of equation (13).

\section{Volume 4 Issue 12, November 2015}




\section{International Journal of Science and Research (IJSR) \\ ISSN (Online): 2319-7064}

Index Copernicus Value (2013): 6.14 | Impact Factor (2014): 5.611

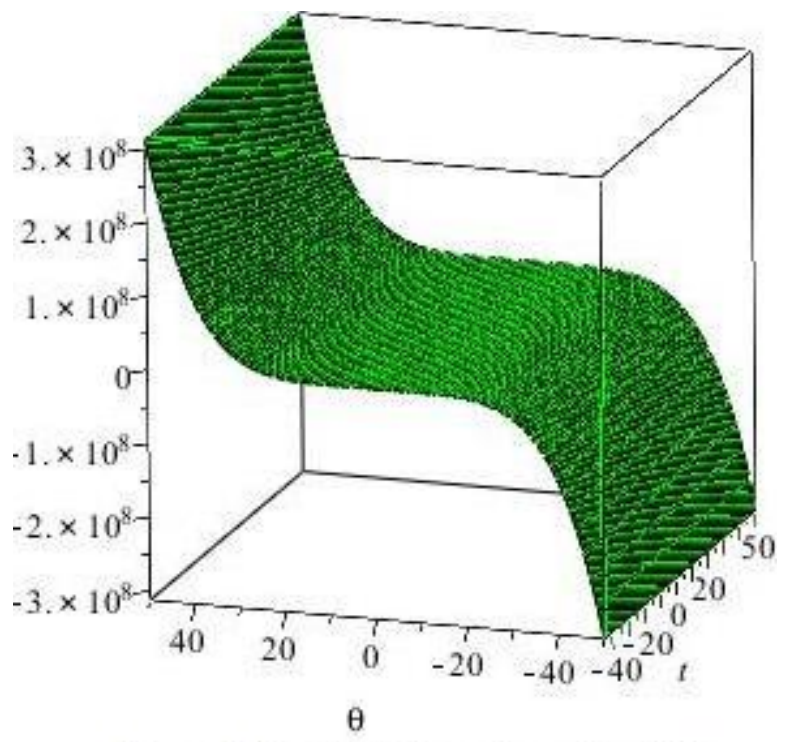

Figure 3: E xact solution of equation (13)

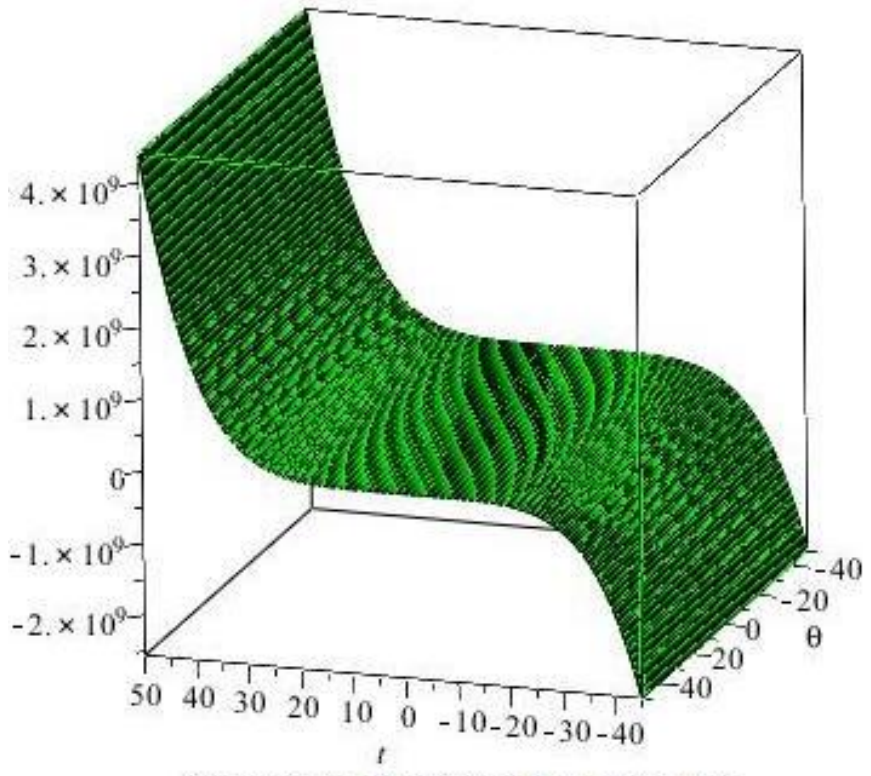

Figure 4: ADM solution of equation (13)

\section{Conclusion}

In this paper, we have successfully applied Adomian Decomposition Method to Bernoulli differential equations. We gave the general Adomian polynomial for the nonlinear term in the Bernoulli differential equations and applied it to practical problems. The problems considered were positive index of the nonlinear term. This can easily be extended to negative index using the general Adomian polynomial of the nonlinear term given in this paper. The result obtained by Adomian Decomposition Method from each Bernoulli differential equations were exactly the same as those of the analytical solutions. And, the exact solutions compared to those obtained by Adomian Decomposition Method are clearly depicted in Figures 1, 2, 3 and 4.

\section{References}

[1] G. Adomian, "Solution of Thomas-Fermi equation", Applied Mathematical Letters, 11(4), pp. 131-133, 1998.
[2] G. Adomian, "Noise Term in Decomposition Solution Series" Computer and Mathematics with Application, 24(11), pp. 61-64, 1992

[3] M. Almazmumy, F. A. Hendi, H. O. Bokodah, and H. Alzumi, "Recent Modification of Adomian Decomposition Method for Initial Value Problems in Ordinary Differential Equations", American Journal of Computational Mathematics, 2, pp. 228-234, (2012).

[4] H. Jafari, and V. Daftardar-Gejji, "Revised Adomian Decomposition Method for Solving Nonlinear Equations", Applied Mathematics and Computation, 175(1), pp. 1-7, (2006).

[5] R. Rach, G. Adomian and R. E. Meryers, "A Modified Decomposition," Computer and Mathematics with Application, 23(1), pp. 19-23, (1992).

[6] H. K. Dash, Advanced Engineering Mathematics, India, S. Chand and Company Ltd, (1999).

[7] S. M. Graham, Differential Equations; Bernoulli Equations, Salford, Promoting Physics Learning and Teaching Opportunities, (2004).

[8] E. U. Agom and A. M. Badmus, "A Concrete Adomian Decomposition Method for Quadratic Riccati's Differential equations", Pacific Journal of Science and Technology, 16(2), pp. 57-62, (2015).

\section{Author Profile}

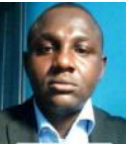

E. U. Agom received B.Sc. and M.Sc. degrees in Mathematics from University of Abuja and Abubakar Tafawa Balewa University in 1997 and 2007 respectively. He is now in Department of Mathematics, University of Calabar, Nigeria. 